\title{
The Algebra of Self-Adjoint Operators in Spectral Theory with Application in Quantum Mechanics
}

\author{
M. Solomon ${ }^{1}$, D. Samaila ${ }^{1}$, S. M. Nengem ${ }^{1}$ \\ ${ }^{1}$ Department of Mathematical Sciences, Adamawa State University, P.O. Box 25, Mubi, Nigeria
}

\begin{abstract}
The main aim of this article is to present the Spectral theory of self-adjoint operators on Hilbert space and to describe its applications in the development of Quantum mechanics. Since in Quantum mechanics, observables correspond to self-adjoint operators, to achieve our aim, we employed the idea of symmetric operators in Hilbert space. We see that there is a bijection between symmetric extensions of an operator and isometric extensions of its Cayley transform. The resolvent $\rho(a)$ is invertible, which is the generalization of the theory of eigenvalues of a matrix, that is, the set of all eigenvalues of an operator is called the spectrum of the operator, and more importantly, the Hermitian operator. Finally, we showed how to determine the energy level of the atom of an element using some derived equations.
\end{abstract}

Keywords: Symmetric operator, Self-adjoint operator, Observables, Eigenvalues, Energy

\section{Introduction}

In mathematics, on a finite-dimensional inner product space, a self-adjoint operator is an operator that is its own adjoint, or, equivalently, one whose matrix is Hermitian, where a Hermitian matrix is one which is equal to its own conjugate transpose. By the finite-dimensional spectral theorem, such operators can be associated with an orthonormal basis of the underlying space in which the operator is represented as a diagonal matrix with entries in $\Re$, the real. In this article, we consider the algebra and importance of Self-adjoint operators in quantum mechanics and their formulation, in which physical observables such as position, momentum, angular momentum and spin are represented by self-adjoint operators on a Hilbert space. Of particular significance is the Hamiltonian

$$
H \psi=V \psi-\frac{\hbar^{2}}{2 m} \nabla^{2} \psi
$$

which as an observable, corresponds to the total energy of a particle of mass $m$ in a real potential field $V$. Differential operators are important classes of unbounded operators.

The structure of self-adjoint operators on infinite-dimensional Hilbert spaces essentially resemble the finitedimensional case, that is to say, operators are self-adjoint if and only if they are unitarily equivalent to realvalued multiplication operators. With suitable modifications, this result can be extended to possibly unbounded operators on infinite-dimensional spaces. Since an everywhere defined self-adjoint operator is necessarily bounded, one needs to be more attentive to the domain issue in the unbounded case.

Also, the absolutely continuous spectrum corresponds to the Free State while the pure points correspond to bounded state (take the momentum and position operator, say). Hence in order to appreciate and apply spectral theory in quantum mechanical setting, we must turn to spectral theory for unbounded operators [2].

The spectral theory of linear operators in Hilbert space is one of the most important tools in the mathematical foundation of quantum mechanics; in fact linear operators and quantum mechanics have had a symbiotic relationship. Quantum mechanics was the profound revolution in Physics. Some very simple systems present nontrivial questions whose answers need a mathematical approach. The theory of linear operators and their spectra constitute a wild field [19]. A linear operator is an operator such that

(i) The domain $\mathcal{D}(T)$ of $T$ is a vector space and the range $\mathcal{R}(T)$ lies in a vector space over the same field,

(ii) For all $x, y \in \mathcal{D}(T)$ and scalar $\alpha, T(x+y)=T(x)+T(y)$ and $T(\alpha x)=\alpha T(x)$.

The first aim in this article is to present the spectral theory of self- adjoint operators on Hilbert space and secondly, show its application in the development of parts of quantum mechanics such as Schrödinger equation, and lastly, to strive to present some examples illustrating concepts and build up confidence with the methods employed. The existence of quantum mechanics is to be equivalent to the self- adjointness of the Hamiltonian operator. In fact, in quantum mechanics, every physical observables are represented by self- adjoint operators on Hilbert space, $H$ ([15]; [12] and [18]). The idea is to complement a text that emphasizes mathematics with additional rigorous approaches to some standard quantum concepts; e.g., why the quantum observables are represented by self-adjoint operators instead of just Hermitian ones. 


\section{Symmetric Operators}

A partially defined linear operator $A$ on a Hilbert space $H$ is called symmetric if

$$
\langle A x \mid y\rangle=\langle x \mid A y\rangle
$$

for all elements $x$ and $y$ in the domain of $A$. More generally, a partially defined linear operator $A$ from a topological vector space $E$ into its continuous dual space $E^{*}$ is said to be symmetric if (2) is satisfied for all elements $x$ and $y$ in the domain of $A$. This usage is fairly standard in the functional analysis literature.

A symmetric everywhere defined operator is self-adjoint. By the Hellinger-Toeplitz theorem, a symmetric everywhere defined operator is also bounded. Bounded symmetric operators are also called Hermitian.

The previous definition agrees with the one for matrices given in the introduction to this article, if we take as $H$ the Hilbert space $\mathbf{C}^{n}$ with the standard dot product and interpret a square matrix as a linear operator on this Hilbert space. It is however much more general as there are important infinite-dimensional Hilbert spaces.

The spectrum of any bounded symmetric operator is real; in particular all its eigenvalues are real, although a symmetric operator may have no eigenvalues.

\section{Self-Adjoint Operators}

Given a densely defined linear operator $A$ on $H$, its adjoint $A^{*}$ is defined as follows:

- The domain of $A^{*}$ consists of vectors $x$ in $H$ such that $y \mapsto\langle x \mid A y\rangle$ (which is a densely defined linear map) is a continuous linear functional. By continuity and density of the domain of $A$, it extends to a unique continuous linear functional on all of $H$.

- By the Riesz representation theorem for linear functionals, if $x$ is in the domain of $A^{*}$, there is a unique vector $z$ in $H$ such that $\langle x \mid A y\rangle=\langle z \mid y\rangle \forall y \in \operatorname{dom} A$.

This vector $z$ is defined to be $A^{*} x$. It can be shown that the dependence of $z$ on $x$ is linear.

Notice that it is the denseness of the domain of the operator, along with the uniqueness part of Riesz representation that ensures the adjoint operator is well defined.

A result of Hellinger-Toeplitz type says that an operator having an everywhere defined bounded adjoint is bounded. The condition for a linear operator on a Hilbert space to be self-adjoint is stronger than to be symmetric.

For any densely defined operator $A$ on Hilbert space, one can define its adjoint operator $A^{*}$. For a symmetric operator $A$, the domain of the operator $A^{*}$ contains the domain of the operator $A$, and the restriction of the operator $A^{*}$ on the domain of $A$ coincides with the operator $A$, i.e. $A \subseteq A^{*}$, in other words $A^{*}$ is an extension of $A$. For a self-adjoint operator $A$, the domain of $A^{*}$ is the same as the domain of $A$, and $A=A^{*}$.

\section{Extensions Of Symmetric Operators}

One may ask a question that: if an operator $A$ on a Hilbert space $H$ is symmetric, does it have selfadjoint extensions? The answer is provided by the Cayley transform of a self-adjoint operator and the deficiency indices. In the symmetric case, the closedness requirement poses no obstacles, since it is known that all symmetric operators are closable. Below are some facts about self-adjoint extensions.

Theorem 1: Suppose $A$ is a symmetric operator. Then there is a unique partially defined linear operator

such that

$$
W(A): \operatorname{ran}(A+i) \rightarrow \operatorname{ran}(A-i)
$$

$$
W(A)(A x+i x)=A x-i x ; \quad x \in \operatorname{dom}(A)
$$

$W(A)$ is isometric on its domain. Moreover, the range of $1-W(A)$ is dense in $H$ [11].

Conversely, given any partially defined operator $U$ which is isometric on its domain (which is not necessarily closed) and such that $1-U$ is dense, there is a (unique) operator $\mathrm{S}(U)$

such that

$$
S(U): \operatorname{ran}(1-U) \rightarrow \operatorname{ran}(1+U)
$$

$$
S(U)(x-U x)=i(x+U x) \quad x \in \operatorname{dom}(U)
$$

The operator $\mathrm{S}(U)$ is densely defined and symmetric. The mappings $\mathrm{W}$ and $\mathrm{S}$ are inverses of each other. The mapping $\mathrm{W}$ is called the Cayley transform. It associates a partially defined isometry to any symmetric densely defined operator. Note that the mappings $\mathrm{W}$ and $\mathrm{S}$ are monotone: This means that if $B$ is a symmetric operator that extends the densely defined symmetric operator $A$, then $\mathrm{W}(B)$ extends $\mathrm{W}(A)$, and similarly for $\mathrm{S}$.

Theorem 2: A necessary and sufficient condition for $A$ to be self-adjoint is that its Cayley transform $\mathrm{W}(A)$ be unitary [11]. 
This immediately gives us a necessary and sufficient condition for $A$ to have a self-adjoint extension, as follows: Theorem 3: A necessary and sufficient condition for $A$ to have a self-adjoint extension is that $\mathrm{W}(A)$ have a unitary extension [11].

Note that a partially defined isometric operator $V$ on a Hilbert space $H$ has a unique isometric extension to the norm closure of $\operatorname{dom}(V)$, while a partially defined isometric operator with closed domain is called a partial isometry.

Theorem 4: A partial isometry $V$ has a unitary extension if and only if the deficiency indices are identical. Moreover, $V$ has a unique unitary extension if and only if both deficiency indices are zero [11].

We see that there is a bijection between symmetric extensions of an operator and isometric extensions of its Cayley transform. An operator which has a unique self-adjoint extension is said to be essentially self-adjoint. Such operators have a well-defined Borel functional calculus. Symmetric operators which are not essentially self-adjoint may still have a canonical self-adjoint extension. Such is the case for non-negative symmetric operators (or more generally, operators which are bounded below). These operators always have a canonically defined Friedrichs extension and for these operators we can define a canonical functional calculus. Many operators that occur in analysis are bounded below (such as the negative of the Laplacian operator), so the issue of essential adjointness for these operators is less critical.

\section{Quantum Mechanics}

Quantum mechanics is the branch of mechanics (science of movement and force) that deals with the mathematical description of the motion on interaction with subatomic particles. It concerns with the description of motion and energy level of microscopic particles such as atoms, molecules, etc. Here, as in any physical theory, we have to consider only those quantities which may be measured. These physical quantities whose values are found by means of an experiment (or measured) are fundamental concept of quantum mechanics called the observables. It turns out to be that, in quantum mechanics it is impossible, in general, to predict exactly the result of measurement [14]. This is a real variable and, in fact, quantum mechanics studies laws of distribution of such variables. Among all observables, there is one of particular importance: the energy. The corresponding operator is called the Hamiltonian, denoted by $H$ or Schrödinger operator

$$
i \hbar \frac{d \psi(t)}{d t}=H \psi(t)
$$

where $H$ is the Hamiltonian and $\hbar$ is the Planck's constant. Also see (1) above.

\section{Self-Adjoint Extensions In Quantum Mechanics}

In quantum mechanics, observables correspond to self-adjoint operators. By Stone's theorem on oneparameter unitary groups, self-adjoint operators are precisely the infinitesimal generators of unitary groups of time evolution operators. However, many physical problems are formulated as a time-evolution equation involving differential operators for which the Hamiltonian is only symmetric. In such cases, either the Hamiltonian is essentially self-adjoint, in which case the physical problem has unique solutions or one attempts to find self-adjoint extensions of the Hamiltonian corresponding to different types of boundary conditions or conditions at infinity.

Example 1. The one-dimensional Schrödinger operator with the potential

$$
V(x)=-(1+|x|)^{\alpha}
$$

defined initially on smooth compactly supported functions, is essentially self-adjoint (that is, has a self-adjoint closure) for $0<\alpha \leq 2$ but not for $\alpha>2$ [7].

Example 2. There is no self-adjoint momentum operator $P$ for a particle moving on a half-line. Nevertheless, the Hamiltonian $P^{2}$ of a "free" particle on a half-line has several self-adjoint extensions corresponding to different types of boundary conditions. Physically, these boundary conditions are related to reflections of the particle at the origin [10].

\section{Spectral Theory}

In mathematics, spectral theory is an inclusive term for theories extending the eigenvalues and eigenvectors of a single square matrix to a much broader theory of the structure of operators in a variety of mathematical spaces [1]. It is a result of studies of linear algebra and the solution of systems of linear equations and their generalizations [20]. The spectral theory is connected to that of analytic function because the spectral properties of an operator are related to analytic functions of the spectral parameter ([3] and [5]).

The starting point of the quantum mechanical formalism is the Hilbert space. In mathematical concepts, it is a space in the sense that it is a complex vector space which is endowed by an inner or scalar product. In quantum mechanics, every physical observable can be described by a self-adjoint operator- an infinite dimensional 
symmetric matrix $[(\mathrm{T} x, y)]=(x, \mathrm{~T} y)$. For example, $-\Delta$ is a self-adjoint operator in $\mathrm{L}^{2}\left(\Re^{d}\right)$ when defined in an appropriate domain. In quantum mechanics, it corresponds to a free particle (just travelling in space). Furthermore, the position of a particle is described by a wave function $\phi(x, t) \in \mathrm{L}^{2}\left(\Re^{d}\right)$. The physical meaning of the wavefunction is that the probability to find it in a region $\Omega$ at time $t$ is equal to $\int|Q(x, t)| d x$. The space of $\mathrm{C}^{n}$ of all $n$-tuple of complex numbers becomes a Hilbert space with inner product. A further requirement in the definition of a Hilbert space is that every Cauchy sequence must be convergent, that is, the space is complete. Quantum mechanics provides a mathematical description of much of the dual particle-like and wavelike behaviour and interactions of energy and matter is based on the postulate that the phase space is a Hilbert space $H$ with the additional postulates that:

(i) Only vectors of norm 1 corresponds to physical states;

(ii) Vectors differing by a phase, i.e., by a complex number of modulus 1, correspond to the same physical state. In other word, $\psi \in H$ and $z \psi$ with $z \in C$ and $|z|=1$ give the same state [12].

\section{Operators On Hilbert Spaces}

The important operators on Hilbert spaces are bounded and unbounded operators. For the purpose of this article much emphasis is given to the unbounded operators with little to the bounded operators. This is because the observables that constitute quantum mechanics are unbounded operators.

8.1 Bounded operators. The operator $T$ is said to be bounded if there is a real number $c$ such that for all $x \in \mathcal{D}(T) \ni\|T x\| \leq c\|x\|$, otherwise unbounded, where $T: \mathcal{D}(T) \rightarrow Y$ is a linear operator, with $\mathcal{D}(T) \subset X$ and $X$ and $Y$ are normed spaces [6].

8.2 Unbounded operators. Unbounded operators are also tractable in Hilbert spaces, and have important applications to quantum mechanics. An unbounded operator $T$ on a Hilbert space $H$ is defined as a linear operator whose domain $\mathcal{D}(T)$ is a linear subspace of $H$. Often the domain $\mathcal{D}(T)$ is a dense subspace of $H$, in which case $T$ is known as a densely defined operator. Self- adjoint unbounded operators play the role of the observables in the mathematical formulation of quantum mechanics. Examples of self- adjoint unbounded operators on the Hilbert space $L^{2}(R)$ are:

(i) A suitable extension of the differential operator: $(A f) f(x)=i \frac{d}{d x} f(x)$, where $i$ is the imaginary unit and $f$ is a differentiable function of compact support.

(ii) The multiplication- by- $x$ operator: $(B f)(x)=x f(x)$.

These correspond to the momentum and position observables respectively.

Unbounded linear operators occur in many applications notably in connection with differential equations and in quantum mechanics. In fact, the theory of unbounded operators was stimulated by an attempt to put quantum mechanics on a rigorous mathematical foundation [9].

\section{Resolvent And Spectrum}

Let $a: D(a) \rightarrow H$ be an (possibly unbounded) operator on a Hilbert space. The resolvent $\rho(a)$ is the set of $z \in C$ for which $a-z: D(a) \rightarrow H$ is injective and surjective (i.e., invertible). The spectrum $\sigma(a):=C / \rho(a)$. The theory of the spectrum of a closed operator on a Hilbert space (which may be bounded or unbounded) is a generalization of the theory of eigenvalues of a matrix. This statement is supported by the following theorem:

Theorem 5: (Spectrum Closed) The resolvent set $\rho(T)$ of a bounded linear operator $T$ on a complex plane $X$ is open. Hence the spectrum $\sigma(T)$ is closed [6].

Proof: If $\rho(T)=0$, then it is close. Let $\rho(T) \neq 0$. For a fixed point $\lambda_{0} \in \rho(T)$ and $\lambda \in C$, we have

$$
\begin{aligned}
T-\lambda I & =T-\lambda_{0} I-\left(\lambda-\lambda_{0}\right) I \\
& =\left(T-\lambda_{0} I\right)\left[I-\left(\lambda-\lambda_{0)}\right)\left(T-\lambda_{0}\right)^{-1}\right]
\end{aligned}
$$

Denoting the operator in the bracket $[\ldots]$ by $V$ we can write this in the form $T_{\lambda}=T_{\lambda_{0}} V$ where

$$
V=I-\left(\lambda-\lambda_{0}\right) R_{\lambda_{0}}
$$

Since $\lambda_{0} \in \rho(T)$ and $\mathrm{T}$ is bounded, it implies that $R_{\lambda_{0}}=T_{\lambda_{0}}{ }^{-1} \in B(X, X)$

Now we show that $\mathrm{V}$ has inverse 


$$
V^{-1}=\sum_{j=0}^{\infty}\left[\left(\lambda-\lambda_{0}\right) R_{\lambda_{0}}\right]^{j}=\sum_{j=0}^{\infty}\left(\lambda-\lambda_{0}\right)^{j} R_{\lambda_{0}}{ }^{j}
$$

In $\mathrm{B}(X, X)$ for all $\lambda$ such that $\left\|\left(\lambda-\lambda_{0}\right) R_{\lambda_{0}}\right\|<1$ that is

$$
\left|\lambda-\lambda_{0}\right|<\frac{1}{\left\|R_{\lambda_{0}}\right\|}
$$

Since $T_{\lambda_{0}}{ }^{-1}=R_{\lambda_{0}} \in B(X, X)$. We see from this and (3) that for every $\lambda$ satisfying (4) the operator $T_{\lambda}$ has an inverse

$$
R_{\lambda}=T_{\lambda}^{-1}=\left(T_{\lambda_{0}} V\right)^{-1}=V^{-1} R_{\lambda_{0}}
$$

Hence (4) represents a neighbourhood $\lambda_{0}$ consisting of regular value $\lambda$ of $T$. Since $\lambda_{0} \in \rho(T)$ was arbitrary, $\rho(T)$ is open so that its complement $\delta(T)=C-\rho(T)$ is closed.*

Theorem 6: (Eigenvalue, Eigenvector) Let $\mathrm{T}: H \rightarrow H$ be a bounded self - adjoint operator on a Hilbert space $H$; then
a. all eigenvalues are real;
b. eigenvectors corresponding to different eigenvalues $\lambda \neq \lambda^{\prime}$ are orthogonal [4].

\section{Proof:}

a. Let $\lambda$ be any eigenvalue of $\mathrm{T}$ and $x$ corresponding eigenvector. Then $x \neq 0$ and $\mathrm{T} x=\lambda x$. Using selfadjointness of $\mathrm{T}$, we obtained $\lambda\langle x, x\rangle=\langle\lambda x, x\rangle=\bar{\lambda}\langle x, x\rangle$. Hence $\langle x, x\rangle=\|x\|^{2} \neq 0$, since $x \neq 0$ and dividing both sides by $\langle x, x\rangle$ gives $\lambda=\bar{\lambda}$. Hence $\lambda$ is real.

b. Let $\lambda$ and $\mu$ be eigenvalues of $\mathrm{T}$ and let $x$ and $y$ be corresponding eigenvectors. Then $\mathrm{T} x=\lambda x$ and $\mathrm{T} y=\mu y$ since $\mathrm{T}$ is self-adjoint and $\mu$ is real.

$$
\lambda\langle x, y\rangle=\langle\lambda x, y\rangle=\langle T x, y\rangle=\langle x, T y\rangle=\langle x, \mu y\rangle=\mu\langle x, y\rangle
$$

Since $\lambda \neq \mu$ we must have $\langle x, y\rangle=0$ which means orthogonality of $x$ and $y$.*

\section{The Time-Independent Schrödinger Equattion}

Spectral theory played a great role in the separation of space and time in Schrödinger equation of quantum mechanics through the derivation of (time-independent) Schrödinger equation [17]. We use the analogy between light waves and de Broglie's matter waves for the derivation of the equation. This is first started with the one- dimensional wave equation

$$
\frac{\partial^{2} \mu}{\partial x^{2}}=\frac{1}{V^{2}} \frac{\partial^{2} \mu}{\partial t^{2}}
$$

This equation is used for investigating refraction, interference and other more subtle optical phenomena in quantum mechanics. By introducing the separation of variables $\mu(x, t)=\psi(x) f(t)$, we have

$$
f(t) \frac{d^{2} \psi(x)}{d x^{2}}=\frac{1}{V^{2}} \psi(x) \frac{d^{2} f(t)}{d t^{2}} .
$$

If we introduce one of the standard wave equation solution for $f(t)$, such as $e^{i \omega t}$, we have

$$
\frac{d^{2} \psi(x)}{d x^{2}}=\frac{-\omega^{2}}{V^{2}} \psi(x)
$$

We now have an ordinary differential equation describing the spatial amplitude of the matter wave as a function of position. The energy of a particle is the sum of kinetic and potential parts $E=\frac{p^{2}}{2 m}+V(x)$, which can be solved for the momentum $p$ to obtain $p=\{2 m[E-V(x)]\}^{1 / 2}$. Now we can use the de Broglie formula to get the expression for the wavelength 


$$
\lambda=\frac{h}{p}=\frac{h}{\{2 m[E-V(x)]\}^{1 / 2} .} .
$$

The term $\frac{\omega^{2}}{V^{2}}$ in (6) can be rewritten in terms of $\lambda$, that is, if we recall that $\omega=2 \pi V$ and $V \lambda=v$ then

$$
\frac{\omega^{2}}{V^{2}}=\frac{4 \pi^{2} V^{2}}{V^{2}}=\frac{4 \pi^{2}}{\lambda^{2}}=\frac{2 m[E-V(x)]}{\hbar^{2}} .
$$

When this result is substituted in (6), we obtain the famous time-independent Schrödinger equation

$$
\frac{d^{2} \psi(x)}{d x^{2}}+\frac{2 m}{\hbar^{2}}[E-V(x)] \psi(x)=0
$$

This is almost always written in the form

$$
\frac{-\hbar^{2}}{2 m} \frac{d^{2} \psi(x)}{d x^{2}}+V(x)=E \psi(x)
$$

This single particle one-dimensional equation can easily be restricted to the case of three- dimensional, where it becomes

$$
-\frac{\hbar^{2}}{2 m} \nabla^{2} \psi(x)+V(r) \psi(r)=E \psi(r)
$$

10.1 The Total Energy Of Hydrogen Atom: As explained earlier by [16], hydrogen atom consists of nucleus with positive charge $+\mathrm{z}_{\mathrm{e}}$, atomic number $\mathrm{z}$ and a single electron. The potential energy of a two- particle system with charges $\mathrm{q}_{1}$ and $\mathrm{q}_{2}$ is given by

$$
V=\frac{1}{4 \pi E_{0}} \frac{q_{1} q_{2}}{r}
$$

Where, $\mathrm{r}$, is the distance between the particles. For the two-particle system of a hydrogen atom, where $q_{I}=+e$ and $q_{2}=-e$, we can write the potential energy as

$$
V=\frac{1}{4 \pi E_{0}} \frac{(e)(-e)}{r}=-\frac{1}{4 \pi E_{0}} \frac{e^{2}}{r}
$$

which is the potential energy function of hydrogen atom.

To find the energies of the quantum states of hydrogen atom, we must solve Schrödinger's equation with (10) substituted for $\mathrm{V}$ in (7) or (8). However, because the electron in the hydrogen atom is trapped in a threedimensional well, we must use a three-dimensional form of Schrödinger equation, i.e. (9). Solving the equation reveals that the energies of the electron quantum states of hydrogen atoms are given by

$$
E_{n}=-\frac{m e^{4}}{8 E_{0} h^{2}} \frac{1}{n^{2}}, \quad n=1,2, \ldots
$$

This is the energy an electron of hydrogen atom can have when an electron is trapped by it. Energy level diagram can be drawn for every atom. That for hydrogen atom is shown by [8]. In this case the lowest level has energy $-13.6 \mathrm{eV}$ and is the one normally occupied by its single electron. Above this state are the excited states to which the hydrogen atom may raise by absorbing the correct amount of energy. If the energy absorbed is sufficient to allow the electron to escape from the atom, the latter becomes ionized; for hydrogen the ionization energy is $13.6 \mathrm{eV}$.

\section{Discussion}

Several importance of self-adjoint operators follow from our definition. First of all, the eigenvalues of self-adjoint operator $T$ are real, as indicated by theorem 6 and its prove, which tells us that the eigenvalues of $T$ corresponding to different eigenvectors are orthogonal. The crucial features of spectral theory are the eigenvalues and eigenvectors which are solutions to some operators. The set of all eigenvalues of an operator is called the spectrum of the operator. The important types of operators in quantum mechanics are Hermitian (selfadjoint) operators. They are of paramount important in quantum mechanics because an observable such as momentum is represented by a Hermitian operator and the possible results of the measurement of this observable are the eigenvalues of the operator (i.e., the eigenvalues are real since the operators are Hermitian). Some formulae for finding the energy of hydrogen atom were derived which can be applied in the calculation of the energy levels in the atom. For instance, to determine the energy of an electron in the ground state of 
hydrogen atom, giving the following information as demonstrated by [16], i.e. $E_{0}=8.85 \times 10^{-12} C^{2} / N M^{2}$, $m=9.1 \times 10^{-31} \mathrm{~kg}, h=6.6 \times 10^{-34} \mathrm{JS}, e=1.6 \times 10^{-19} \mathrm{C}$, we have, by direct substitution in equation 11 above with $n=1$,

$$
E_{1}=-\frac{\left(9.1 \times 10^{-31} \mathrm{~kg}\right)\left(1.6 \times 10^{-19} \mathrm{C}\right)^{4}}{8\left[8.85 \times 10^{-12}\left(C^{2} / N . M^{2}\right)\right]^{2}(1)^{2}\left(6.63 \times 10^{-34}\right)^{2}}=-2.17 \times 10^{-18} \mathrm{~J} .
$$

The energy levels can be calculated for different elements. The energy of an atom when it is in the $\mathrm{n}^{\text {th }}$ state can be found with the help of the time- independent Schrödinger equation in three-dimensional space. For a nucleus with charge $Z_{e}$ orbited by a single electron, the corresponding relations are

$$
r_{n}=(0.0053 n m)\left(\frac{n^{2}}{z}\right) \text { and } E_{n}=\frac{-13.6 Z^{2}}{n^{2}} \mathrm{eV}
$$

Wave mechanics permits the electron in an atom to have only certain energy values. These values are called the energy levels of the atom.

\section{Conclusion}

Spectral theory is an important tool in the field of sciences, particularly in Quantum mechanics. The operators in quantum mechanics were described using spectral theory, where the spectral theory simplified the derivation of time-independent Schrödinger equation. We have seen some applications of Spectral theory in finding the energy level of an atom of hydrogen. The equations used in finding the energy level can always be used for all elements. The calculation of energy level of an element can always be achieved by any of (7), (8) or (9). And lastly, quantum mechanics depend on the spectral theory of Hilbert space such as self-adjoint operators; since any observable (e.g. energy, position and momentum) is represented by a Hermitian operator and eigenvalues are the possible results of the momentum of these observables. The eigenvalues are real since the operator is Hermitian.

\section{References}

[1] A. J. Dieodonne, History of functional analysis; Elservier, 1981.

[2] A. Ramsay, A theorem on two commuting observables; Journal of mathematics and mechanics 15 (2), $1966,227-234$.

[3] A. Sadovnichii, The geometry of Hilbert space- the spectral theory of operators; Springer, 1991.

[4] D. Petz, and M. A. Neilson, Simple proof of the sub-additivity; Quantum, int. comp. (5), 2005, 507-513.

[5] E. G. Belterametti, and S. Bugajski, Effective Algebras and statistical physical theory; Inter. J. Theor. Thys. 38(6), 1997, $3020-3030$.

[6] E. Kreyszig, Introductory Functional Analysis with applications; John Wiley and sons ,Inc., New York, 1978.

[7] F. A. Berezin, and M. A. Shubin, The Schrödinger Equation; Kluwer, 1991.

[8] J. B. Frederick, and H. Eugene, Schaum's outlines of theory and problems of College Physics; John Willey and Sons Inc., New York, 2006.

[9] J. R. Boccio, Notes on Hilbert space and quantum mechanics; Smarthmore college printing press, 2012.

[10] M. Reed, and B. Simon, Methods of Mathematical Physics; Vol 2. Academic Press, 1972.

[11] N. I. Akhiezer, and I. M. Glazman, Theory of Linear Operators in Hilbert Space; Two volumes, Pitman, 1981.

[12] N. P. Landsmann, Lecture notes on Hilbert spaces and Quantum mechanics. http://www.math.kun.nl/landsmann/HSQM.html, 2004.

[13] P. Eduard, Quantum mechanics in Hilbert space ( ${ }^{\text {nd }}$ ed.); Dover, 2006.

[14] P. H. Young, and A. R. Freedman, Sears and Zemanaky's University Physics (11 ${ }^{\text {th }}$ ed.); Pearson Education, Inc., One Lake Street, Upper Saddle River, NJ 0745, 2005, U.S.A.

[15] P. Lahti, And S. Pulmannova'a, Coexistence vs. Functional coexistence of quantum observables, Rep. Math. Phy., 47, $2001,199-212$.

[16] R. L. Reece, University Physis; Brooks/cole publishing company; Pacific Grove Ca 93950 U.S.A, 2000, pp.1260-1265.

[17] S. N. Ghashal, Atomic Physics; Sehand and company Ltd, New Delhi, 2008.

[18] S. P. Gudder, Uniqueness properties of bounded observables; Pacic Journal of mathematics, 19(1), 2011, 81-93.

[19] V. C. R. Oliveira, Intermediate Spectral theory and Quantum dynamics. http:/www.uts ar.br/oliveira/ISTbook.html, 2008.

[20] W. Arveson, Spectral theory and Banach algebras; A short course on spectral theory; Springer, 2002. 